\title{
A methodology for low-speed broadband rotational energy harvesting using piezoelectric transduction and frequency up-conversion
}

\author{
Hailing Fu*, Eric M. Yeatman \\ Department of Electrical and Electronic Engineering, Imperial College London, London, SW7 2AZ, United Kingdom
}

\begin{abstract}
Energy harvesting from vibration for low-power electronics has been investigated intensively in recent years, but rotational energy harvesting is less investigated and still has some challenges. In this paper, a methodology for low-speed rotational energy harvesting using piezoelectric transduction and frequency up-conversion is analysed. The system consists of a piezoelectric cantilever beam with a tip magnet and a rotating magnet on a revolving host. The angular kinetic energy of the host is transferred to the vibration energy of the piezoelectric beam via magnetic coupling between the magnets. Frequency up-conversion is achieved by magnetic plucking, converting low frequency rotation into high frequency vibration of the piezoelectric beam. A distributed-parameter theoretical model is presented to analyse the electromechanical behaviour of the rotational energy harvester. Different configurations and design parameters were investigated to improve the output power of the device. Experimental studies were conducted to validate the theoretical estimation. The results illustrate that the proposed method is a feasible solution to collecting low-speed rotational energy from ambient hosts, such as vehicle tires, micro-turbines and wristwatches.
\end{abstract}

Keywords: rotational energy harvesting, piezoelectric, frequency up-conversion, magnetic plucking, low-speed, broadband

\section{Introduction}

Over the last decade, power supplies have become a critical bottleneck for the development of wireless sensor networks due to the limited lifetime of batteries and the requirement of regular replacement or recharging [1,2]. Energy harvesting from ambient wasted energy to generate sustainable electricity for low-power electronic devices is becoming increasingly attractive as an alternative to conventional batteries. By integrating energy harvesting technologies into wireless sensor networks, the networks can operate autonomously with maintenance costs alleviated. In addition, recent advances in ultra-low power electronics [3] also facilitate the idea of self-powered smart sensing using energy harvesting for power supply.

Various types of energy sources, such as heat, light, mechanical motion and fluid flow, are readily available in the places where wireless sensors are installed. Among these sources, vibrational energy has drawn great attention and has been extensively studied for a decade. The progress and research topics on vibration energy harvesting are summarized in several comprehensive review papers, such as vibration energy harvesting from human motion and machine operation in [4,5], nonlinear mechanisms for broadband energy harvesting in [6,7] and functional materials for energy conversion in [8]. However, as another type of kinetic energy, rotational energy is less investigated and harnessed for power supply, but has unique dynamics that would be beneficial to some applications, including tire pressure sensing $[9,10]$, condition monitoring of rotating machines [11,12] and fluid flow energy harvesting using miniature turbines [13].

The great majority of large scale electrical generation uses a continuously rotating electromagnetic machine for transduction, in which power is extracted from the relative motion between a stationary part, anchored in place, and

\footnotetext{
${ }^{*}$ I am the corresponding author.

Email addresses: h.fu14@imperial.ac.uk (Hailing Fu), e.yeatman@imperial.ac.uk (Eric M. Yeatman)
} 
a rotating part coupled to a source of motion such as a turbine. In miniature energy harvesting, the source of motion (the host, e.g. the human body or vibrating machinery) is typically much larger than the intended generator, and so the need to attach the harvester to both the moving body and a stationary base, or to two locations moving relative to each other (e.g. upper and lower leg [14]), places severe constraints on both the degree of miniaturisation and on usable locations.

Instead, if the motion is reciprocating, an inertial mass can be used in place of the fixed anchor, and this has been the basis for a very wide range of harvesters. Although most of these have proof masses that move rectilinearly [5], rotating proof masses are also possible. Pillatsch et al. developed a rotational energy harvester for wearable devices [15]. Rotational motion was induced by human linear motion using an eccentric semi-circular mass pivoting around a central axis. A piezoelectric beam was clamped on the harvester's frame on the human body and plucked by the rotating mass in a non-continuous fashion. This kind of harvester has the advantage of having no fixed endstops, and can be driven by both rotating and rectilinear motion.

While rotating inertial harvesters can be driven by rotating host motion, this motion must be reciprocating, or at least varying in some way, such as in speed or orientation. Manla et al. developed a non-contact piezoelectric rotational energy harvester with centrifugal force as the impacting force [16]. The harvester consisted of a tube, a piezoelectric element mounted at each end of the tube, a magnet fixed on the piezoelectric elements and a central magnet levitated in the middle of the tube. The variation of the centrifugal force on the central magnet and the outer magnets created the changes of the shape of the piezoelectric elements. However, the orientation of the tube was required to be unchanged on a rotating host to achieve the variation of the relative direction between the centrifugal force and the motion of magnets, which requires attachment of part of the device to a non-rotating structure, which greatly limits the application range.

Continuous rotation with constant speed and the generated centrifugal force cannot be used to drive a truly inertial harvester (i.e. one in which the transducer acts on relative motion between the moving host and an inertial mass). However, centrifural force can be used for other purposes, such as frequency tuning, or position adjusting, rather than direct energy harvesting. Gu and Livermore demonstrated a passive self-tuning rotational energy harvester for variable speed situation [17]. This design consisted of two beams arranged radially: a rigid piezoelectric harvesting beam and a soft driving beam with a tip mass. The piezoelectric beam was impacted by the soft beam due to the deformation caused by the gravity of the tip mass. The centrifugal force generated on the tip mass by rotation was utilized to adjust the natural frequency of the driving beam. The natural frequency tracked the rotation frequency over a range. Fu and Yeatman developed a miniaturized turbine for low-speed airflow energy harvesting [18]. The centrifugal force generated by the rotation of a primary magnet was exploited to decrease the cut-in speed of a rotational airflow energy harvester. The magnetic coupling in the harvester was passively regulated by the centrifugal force with regard to airflow speed.

In certain situations, gravity can provide the counter-force for energy harvesting instead of inertial force, since its direction will vary with respect to the centrifugal force on an eccentric mass. Thus the same structure can be used as that of a rotating inertial harvester, although with the restriction that the plane of motion must have a vertical component. Roundy and Tola developed a rotational energy harvester for tire pressure monitoring applications using the gravitational field [19]. An actuator ball was installed inside a curved track on the vehicle rim. The relative motion between the ball and the rim was created by the gravitational field. The ball rolls back and forth along the track with regard to the influence of gravity, impacting two piezoelectric beams fixed alongside the track once per cycle. Similarly, Toh et al. presented a continuous rotational electromagnetic energy harvester using gravitational torque [20]. An off-centred mass was attached on a conventional DC generator to create gravitational torque. This torque generated a relative motion between the generator and its rotating host, and then electric energy was collected from the relative motion.

A final implementation of a miniature rotating harvester is one that uses the motion of fluid, typically air, past a body to which the harvester can be attached. An example is the micro-wind turbine. Perez et al. presented a centimetre-scale electrostatic wind turbine for airflow energy harvesting [21]. The flow motion was converted to the rotation of turbine blades and electrets were mounted on the edges of blades as rotational components. The stationary electrodes were fabricated on the turbine housing. The relative motion in this case was induced by airflow and electrical power was collected via the variation of the capacitance between the rotating electrets and the static electrodes. Fu et al. developed a footstep energy harvester using heel-strike induced rotation [22]. An air bladder was embedded into a shoe cushion to create airflow, and the rotational motion was generated by a miniature turbine with 
a DC generator attached.

Base on the above discussion, we can identify three classes of rotating miniature motion energy harvesters: inertial devices driven by varying (usually reciprocating) motion; continuously rotating devices using gravity as the counter force; and fluid flow turbines. The choice among these types of harvesters depends mainly on practical constraints in particular applications.

In terms of energy transduction for rotational energy harvesting, the dominant mechanisms are piezoelectric $[19,15]$, electromagnetic $[20,23]$ and electrostatic [21]. Their pros and cons are extensively investigated in vibration energy harvesting [10,24]. Electrostatic conversion requires initial charges on the electrodes. Electret-based harvesters [25] provide a solution, in which the material is only charged once during fabrication with a high voltage, but the capacity of harvesting energy is still limited by the magnitude of capacitance that can be achieved and by the charge density of electrets. Electromagnetic transduction uses the relative motion between coils and magnets. This method is widely used in power generation, such as conversion DC generators, but for small scale devices, the performance is constrained by the number of coils, the magnetic flux gradient and the relative velocity between the components. Due to the low output voltage from this conversion, a high energy loss happens during rectification. The piezoelectric approach is a favourable conversion mechanism for its simplicity in structure and high output voltage. However, the brittle characteristic of this material [26] is detrimental to its long stability. Additional considerations are required to protect it from degradation.

The source of rotational energy is also critical for energy harvester design, as the concerns are different for different cases. Compared to the amount of energy collected by harvesters, the rotational energy source can be divided into two categories - unlimited and limited. For unlimited sources, like rotating wheels and machines, the energy provided by the host is tremendous compared with the energy collected by the harvesters, and the rotational motion of the host is not significantly affected by the harvesters, so the main considerations are the amount of energy that can be collected from the source [20], the operating bandwidth [19], and the compactness of the device [15]. For limited sources, such as miniature airflow turbines, the energy stored by the rotating host is modest and the harvesters should exploit the energy to the largest extent. Therefore, the harvesters have an impact on the rotational motion of the rotating host. The efficiency [27, 28], the start-up requirements [18] and the operating bandwidth [29] are the main consideration in this case.

In this paper, we present a generalised methodology for low-speed broadband rotational energy harvesting. A piezoelectric rotational energy harvester using frequency up-conversion is theoretically analysed and experimentally verified in terms of structural configuration, electromechanical behaviour and frequency respond. This study provides an in-depth understanding of the characteristics of this design. This rotational harvester has a wide bandwidth at low frequency, and is feasible to be adapted to most rotational energy harvesting scenarios, such as energy harvesting from human motion, rotating machines or fluid flow.

\section{Design and operating principle}

The schematic of the rotational energy harvester is shown in Fig. 1(a). It consists of a piezoelectric beam, a tip magnet and a driving magnet on a rotating host. The beam is fixed on a stationary structure, and the tip magnet is attached at the beam's free end. The driving magnet is placed underneath the tip magnet on a revolving host.

A non-contact magnetic plucking mechanism is achieved by the magnets. The beam is contactlessly plucked once per cycle by the magnetic force provided by the driving magnet. This mechanism avoids the situation of an overstressed piezoelectric beam caused by direct impacts [30,31]. Considering the brittle characteristic of piezoelectric materials, the contactless plucking enhances the reliability of the device by reducing the possibility of the degradation of the materials.

Another special character of the harvester is the implementation of frequency up-conversion, which allows the harvester to effectively operate in broadband, low-frequency applications. For conventional inertial piezoelectric harvesters, most have been resonant narrowband devices, often operating at high frequency (100's of $\mathrm{Hz}$ to $\mathrm{kHz}$ ) $[32,33]$ and therefore are not suitable to practical low-frequency broadband applications. The limitation is a direct consequence of the low electromechanical coupling coefficients of piezoelectric transducers. Their output impedance is dominated by the internal capacitance that cannot be tuned out with an inductor of realistic size and efficiency. As a result the electrical circuit cannot effectively damp the mechanical oscillation to achieve a low $Q$. This explains why piezoelectric transducers normally have a narrow bandwidth at high frequency. 
(a)

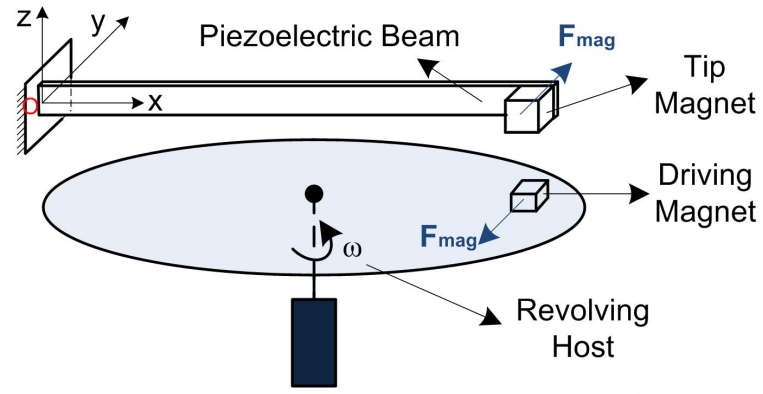

(b)

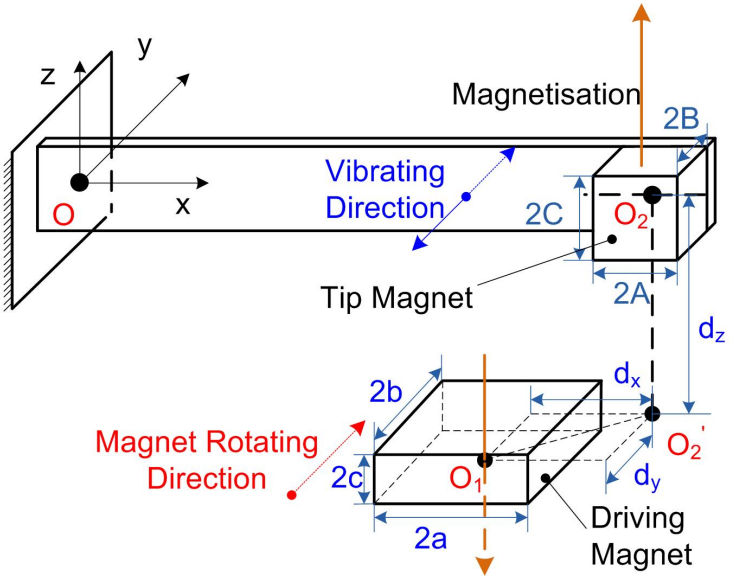

Figure 1: (a) Schematic of the rotational energy harvester and (b) Configuration of the magnetic coupling and structural parameters.

Frequency up-conversion is a mechanism allowing the piezoelectric transducers to operate at their resonant frequency from any low-frequency motion. This mechanism has been widely used in vibration energy harvesting [34, 35]. In this paper, the mechanism is implemented by magnetic plucking of the piezoelectric beam by the rotating driving magnet. Due to the high Q (high resonant frequency and low electrical damping) of the beam, it can still be vibrating from the preceding plucking. The beam oscillates at its natural frequency with an exponential decay, before the second plucking appears. Therefore, the low rotation frequency of the host is converted to the high vibration frequency of the piezoelectric beam. This technique allows the system to exhibit a wide bandwidth, so that the system is capable of harnessing low-speed rotational energy over a wide range.

\section{Modelling and analysis}

\subsection{Modelling of the magnetic coupling}

The magnetic force exerted on the piezoelectric beam is critical to the performance of the energy harvester. In order to analyse and to optimize the magnetic coupling, a theoretical model is built according to the theory developed by Akoun and Yonnet [36]. The configuration and structural parameters of the magnetic coupling is presented in Fig.1(b).

The magnetic force between two cuboidal magnets in the beam vibrating direction can be calculated using

$$
F_{\text {mag }}^{y}=\frac{J \cdot J^{\prime}}{4 \pi \mu_{0}} \sum_{i=0}^{1} \sum_{j=0}^{1} \sum_{k=0}^{1} \sum_{l=0}^{1} \sum_{p=0}^{1} \sum_{q=0}^{1}(-1)^{i+j+k+l+p+q} \cdot \phi_{y},
$$

where $J$ and $J^{\prime}$ are the magnetization of the magnets, $\mu_{0}$ is the magnetic constant, and $\phi_{y}$ is a function of the magnet dimensions and their gaps in 3 axes. The function is given by

$$
\phi_{y}=\frac{1}{2}\left(U_{i j}^{2}-W_{p q}^{2}\right) \ln \left(r-V_{k l}\right)+\frac{1}{2} r V_{k l}+U_{i j} W_{p q} \tan ^{-1}\left(\frac{U_{i j} V_{k l}}{r W_{p q}}\right)+U_{i j} V k l \ln \left(r-U_{i j}\right),
$$


where

$$
\begin{aligned}
& U_{i j}=d_{x}+(-1)^{j} A-(-1)^{i} a, \\
& V_{k l}=d_{y}+(-1)^{l} B-(-1)^{k} b, \\
& W_{p q}=d_{z}+(-1)^{q} C-(-1)^{p} c, \\
& r=\sqrt{U_{i j}^{2}+V_{k l}^{2}+W_{p q}^{2}} .
\end{aligned}
$$

These lengths, $U_{i j}, V_{k l}$ and $W_{p q}$, correspond to the distance between the cube corners and their projections on the axes. The parameters $i, j, k, l, p$ and $q$, are equal to 0 or 1 according to the specific corner. $d_{x}, d_{y}$ and $d_{z}$ are the gaps between the two magnets in three axes. Assuming the rotational frequency of the host is $\omega_{h}$, then the gaps can be expressed as

$$
\begin{array}{r}
d_{x}=r_{m}+d_{x 0}-r_{m} \cos \left(\omega_{h} t-\alpha_{m 0}\right), \\
d_{y}=r_{m} \sin \left(\omega_{h} t-\alpha_{m 0}\right)-\omega(L, t), \\
d_{z}=d_{z 0},
\end{array}
$$

where $d_{x 0}$ and $d_{z 0}$ are the initial gaps along the $\mathrm{x}$-axis and z-axis when the rotor is static, with the driving magnet's angular position $\alpha_{m 0}=0, \omega(L, t)$ is the tip displacement of the piezoelectric beam in the y direction, namely the beam bending direction, and $r_{m}$ is the rotational radius of the driving magnet.

\subsection{Modelling of the piezoelectric beam}

Lumped parameter modelling and distributed parameter modelling are two dominant analytical modelling methods for the dynamic analysis of piezoelectric beams. The lumped parameter model describes the dynamics of a certain point by calculating the equivalent mass, stiffness and damping of a beam to the concerned point $[24,37]$. The process of equivalence limits the model to a single-degree-of-freedom system, and a correction factor is required when the tip mass is considered at the beam's free end [38]. Compared with the lumped parameter model, the distributed parameter model has higher accuracy by avoiding the process of equivalence and by considering the structure as a series of infinitely small elements in a continuous fashion [38, 39].

In this study, the distributed parameter model is used according to the work established by Erturk and Inman $[38,40]$. Adaptation has been made to the rotational energy harvester model in terms of the excitation method. The direct-force load at the beam's free end is used instead of base excitation. The analysis is based on the case of a bimorph connected in series with a load resistance, as shown in Fig. 2.

The dynamic equation of the piezoelectric beam under magnetic plucking at the free end can be written as

$$
Y I \frac{\partial^{4} \omega(x, t)}{\partial x^{4}}+c_{s} I \frac{\partial^{5} \omega(x, t)}{\partial^{4} x \partial t}+c_{d} \frac{\partial \omega(x, t)}{\partial t}+m \frac{\partial^{2} \omega(x, t)}{\partial t^{2}}-\vartheta v(t)\left[\frac{d \delta(x)}{d x}-\frac{d \delta(x-L)}{d x}\right]=F_{\text {mag }}^{y}(t) \delta(x-L),
$$

and

$$
\frac{C_{p}}{2} \frac{d v(t)}{d t}+\frac{v(t)}{R_{l}}+\bar{e}_{31} h_{t} b \int_{0}^{L} \frac{\partial^{3} \omega(x, t)}{\partial x^{2} \partial t} d x=0,
$$

where $Y I$ is the bending stiffness, $\omega(x, t)$ is the transverse deformation of the beam, $c_{s} I$ is the internal damping, $c_{d}$ is the viscous deformation damping, $m$ is the mass per unit length of the beam, $\delta(x)$ is the Dirac delta function, $\vartheta$ is the piezoelectric coupling term in physical coordinates, $v(t)$ is the voltage across a resistive load $R_{l}, C_{p}$ is the inherent capacitance of the piezoelectric beam, $\bar{e}_{31}$ is the piezoelectric constant, $h_{t}$ is $\left(h_{p}+h_{s}\right) / 2, b$ is the width of the beam and $L$ is the length of the beam. 


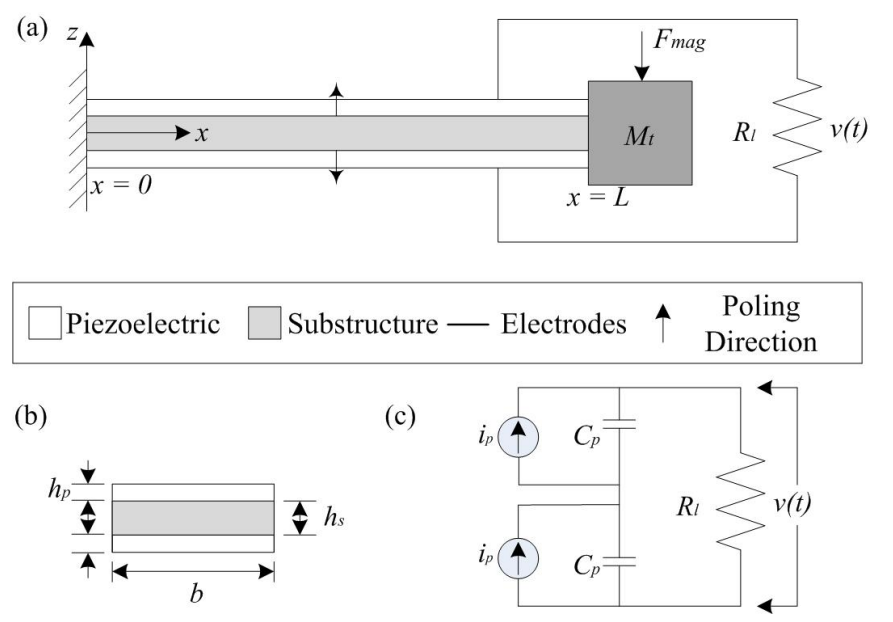

Figure 2: Schematic of the piezoelectric beam. (a) Bimorph piezoelectric beam configuration with the series connection, (b) the cross-sectional view and (c) the equivalent circuit.

\subsection{Arrangements of magnets and piezoelectric beam}

In order to maximize the input power from the revolving host to the piezoelectric cantilever, the arrangements of magnetic coupling and the beam orientation are investigated in terms of the beam bending direction, the magnet rotation direction, the magnetization direction and the initial relative position of the two magnets. Four potentially achievable arrangements are chosen as shown in Fig. 3. In all cases, the cantilever is oriented along the x-axis direction and the beam bending direction is along the y-axis. There are other possible configurations, but they are either equivalent or inferior compared with the illustrated four configurations. The details about the configurations are described below.

(a) The beam bending direction (y-axis) is parallel to the magnetic rotation direction (y-axis) and perpendicular to the magnetization direction (z-axis). The magnet rotation direction is perpendicular to the magnetization direction. The plane composed by the centroids of the two magnets and the magnet rotation direction is parallel to the $y-z$ plane.

(b) The beam bending direction (y-axis) is perpendicular to the magnet rotation direction (x-axis) and the magnetization direction (z-axis). The magnet rotation direction is perpendicular to the magnetization direction. The plane composed by the centroids of the two magnets and the magnet rotation direction is the $x-y$ plane.

(c) The beam bending direction (y-axis) is parallel to the magnet rotation direction (y-axis) and the magnetization direction (y-axis). The magnet rotation direction is in parallel with the magnetization direction. The plane composed by the centroids of the two magnets and the magnet rotation direction is parallel to the $\mathrm{y}-\mathrm{z}$ plane.

(d) The beam bending direction (y-axis) is perpendicular to the magnet rotation direction ( $\mathrm{x}$-axis) and parallel to the magnetization direction (y-axis). The magnet rotation direction is parallel to the magnetization direction. The plane composed by the centroids of two magnets and the magnet rotation direction is the $\mathrm{x}-\mathrm{y}$ plane.

The magnetic force for each configuration is calculated using the theory provided above. The design parameters of the harvester are summarized in Table 1. In order to validate the theoretical model, the finite element method (FEM) is also adopted to compare with the theoretical results.

The results for specific configurations are listed in Table 2. The theoretical results are close to the results from FEM with errors less than $3 \%$. This verifies the validity of the theoretical model for further analysing the performance of different arrangements. The magnetic force in the beam bending direction against the angular position of the rotating magnet for different arrangements are illustrated in Fig. 4. For arrangement (a) and (c), the direction of magnetic force is changing during each excitation cycle, but the direction for arrangement (b) and (d) is almost uniform during 
(a)

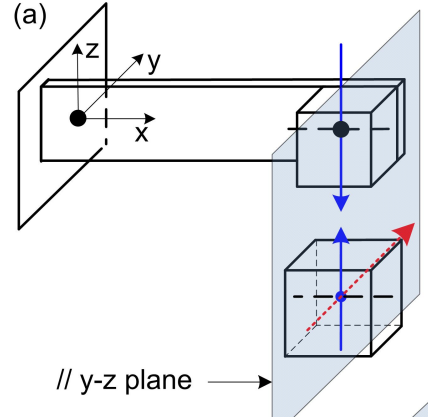

(c)

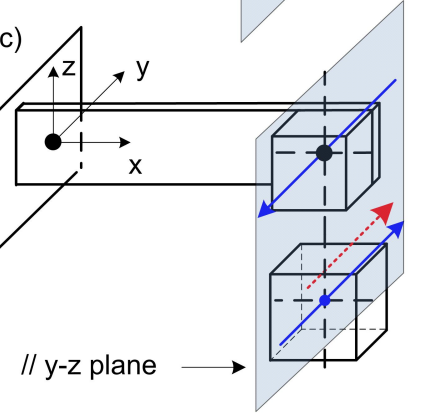

Rotational Direction (b)

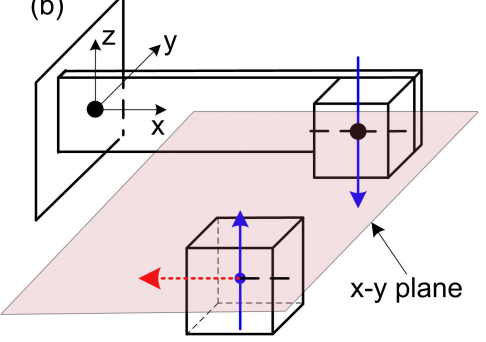

(d)

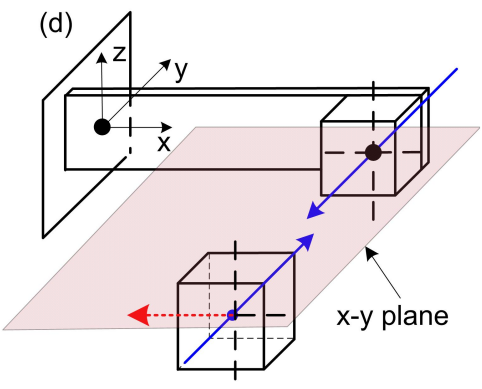

Figure 3: Arrangements of the magnetic coupling in terms of magnetization direction, beam bending direction and magnetic moving direction.

the whole excitation period. The magnetic force for arrangement (d) is much higher than that for other arrangements, which shows the potential option for the rotational energy harvester.

The average output power of the piezoelectric transducer is determined by the input power rather than the input magnetic force. In order to determine the best arrangement, the input power is calculated using the magnetic force and the vibration velocity of the piezoelectric beam. The average input power is given as follows:

$$
P_{a v g}=\frac{\int_{t_{0}}^{t_{1}} F_{m a g}^{i} \cdot \dot{\omega}(L, t) d t}{t_{1}-t_{0}},
$$

where $F_{\text {mag }}^{i}$ is the magnetic force in the beam bending direction $(i$-axis, $i=x, y, z)$ and $\dot{\omega}(L, t)$ is the transverse velocity of the piezoelectric beam's free end. $t_{0}$ and $t_{1}$ are the time considered for averaging. The duration should be long enough to alleviate the influence of the variation of the input power during one cycle. In this calculation, one full rotation cycle is considered for averaging.

Fig. 5 shows the beam tip velocity, the magnetic force in the beam bending direction and the input power for

Table 1: Design parameters of the piezoelectric harvester.

\begin{tabular}{lcr}
\hline Symbol & Description & Value \\
\hline$L \times b$ & Beam size & $26.5 \mathrm{~mm} \times 1.5 \mathrm{~mm}$ \\
$h_{p}$ & Thickness of piezo layer & $0.1 \mathrm{~mm}$ \\
$h_{s}$ & Thickness of substrate & $0.1 \mathrm{~mm}$ \\
$r_{m}$ & Magnet rotation radius & $12 \mathrm{~mm}$ \\
$a \times b \times c$ & Driving magnet size & $1.5 \times 1.5 \times 1.5 \mathrm{~mm}^{3}$ \\
$A \times B \times C$ & Tip magnet size & $0.5 \times 0.5 \times 0.5 \mathrm{~mm}^{3}$ \\
$d_{z 0}$ & Initial gap in z-axis & $3.2 \mathrm{~mm}$ \\
$J$ & Magnetization of magnets & $1.17 \mathrm{~T}$ \\
$\rho_{m}$ & Density of magnets & $7400 \mathrm{~kg} / \mathrm{m}^{3}$ \\
$\bar{e}_{31}$ & Piezoelectric constant & $-22.2 \mathrm{~V} \cdot \mathrm{m} / \mathrm{N}$ \\
\hline
\end{tabular}


Table 2: Peak Magnetic force with different arrangements using theoretical and simulation method

\begin{tabular}{cccccccc}
\hline Arrangement & $\begin{array}{c}\text { Theory } \\
{[\mathrm{mN}]}\end{array}$ & $\begin{array}{c}\text { FEM } \\
{[\mathrm{mN}]}\end{array}$ & Error & $\begin{array}{c}\text { Initial Gaps [mm] } \\
(\mathrm{dx}, \mathrm{dy}, \mathrm{dz})\end{array}$ & $\begin{array}{c}\text { Beam Bending } \\
\text { Direction }\end{array}$ & $\begin{array}{c}\text { Magnetization } \\
\text { Direction }\end{array}$ & $\begin{array}{c}\text { Magnet Rotation } \\
\text { Direction }\end{array}$ \\
\hline (a) & 7.24 & 7.20 & $0.55 \%$ & $(0,0,5)$ & Y - Axis & Z - Axis & Y - Axis \\
(b) & 7.73 & 7.51 & $2.85 \%$ & $(0,5,0)$ & Y - Axis & Z - Axis & X - Axis \\
(c) & 5.12 & 5.24 & $2.29 \%$ & $(0,5,0)$ & Z - Axis & Y - Axis & Z - Axis \\
(d) & 15.45 & 15.38 & $0.45 \%$ & $(0,0,5)$ & Z - Axis & Y - Axis & X - Axis \\
\hline
\end{tabular}

(a)

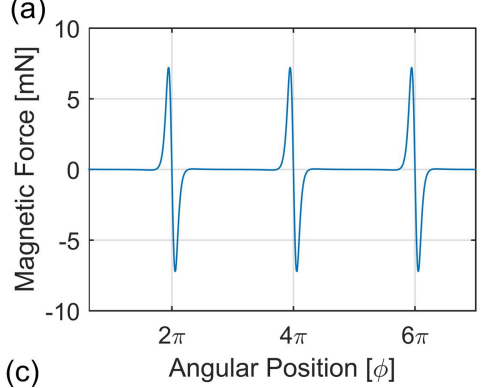

(c)

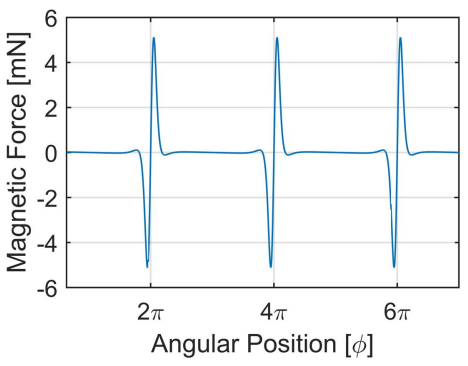

(b)

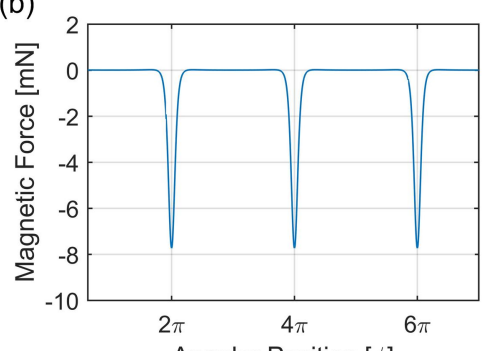

(d)

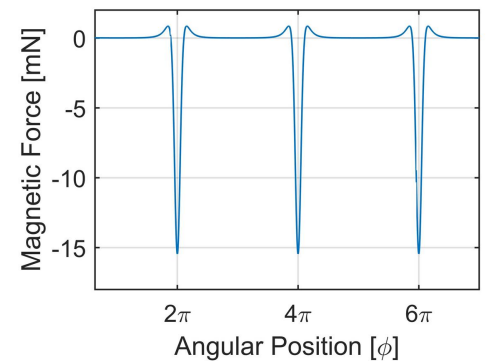

Figure 4: Theoretically achieved magnetic force in the beam bending direction against angular position of the rotating magnet for different arrangements.

different magnetic field arrangements with the excitation frequency of $30 \mathrm{~Hz}$. As illustrated, the input power is determined by both the magnetic force and the beam tip velocity. When their phases are the same, the work is done on the piezoelectric beam. However, the energy is extracted from the piezoelectric beam by the rotating magnet, when the magnetic force direction is opposite to the direction of beam tip velocity.

The phase difference between the magnetic force and the beam tip velocity is mainly caused by the different operating frequencies of the vibrational beam and the rotating magnet. In this design, the beam is under a pulse excitation by the magnetic force and works in a damped-free-vibration form, in which the operating frequency of the beam is its natural frequency. The frequency variation of the driving magnet generates the fluctuation of the phase difference, leading to the variation of the input energy per excitation cycle. Hence, although the first arrangement in Fig. 5(a) has a higher input power at $30 \mathrm{~Hz}$, it is worth investigating the dependence of the input power on frequency in detail.

First, the achievable energy by the piezoelectric beam per excitation cycle is calculated against rotational frequency of the driving magnet, as shown in Fig. 6. The harvester has a wide bandwidth compared to conventional vibration energy harvesters. Meanwhile, the input energy has significant variations with regard to different excitation frequencies for different arrangements. The general trend is upward with ripples when the frequency is high. At low frequencies (below $30 \mathrm{~Hz}$ ), arrangement (a) has the highest input energy; at high frequencies (above $30 \mathrm{~Hz}$ ), arrangement (d) is the best.

The ripples of the input energy at high frequencies are caused by the interaction between the beam vibration generated by two excitations, as shown in Fig. 7. When the excitation frequency is low (20 Hz in Fig. 7(a)), the beam 
(a)

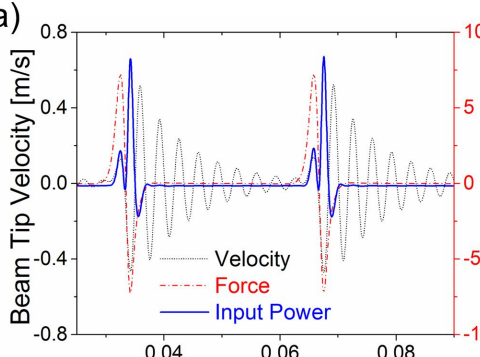

(c)

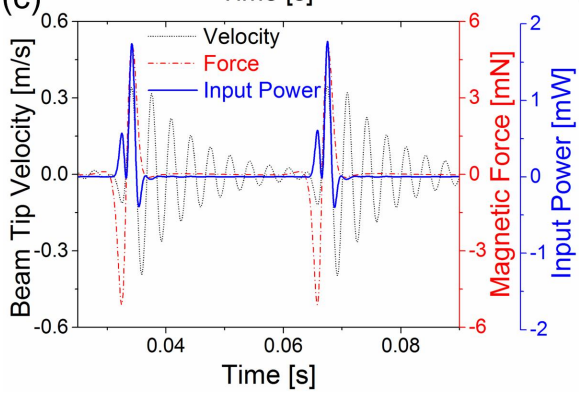

(b)

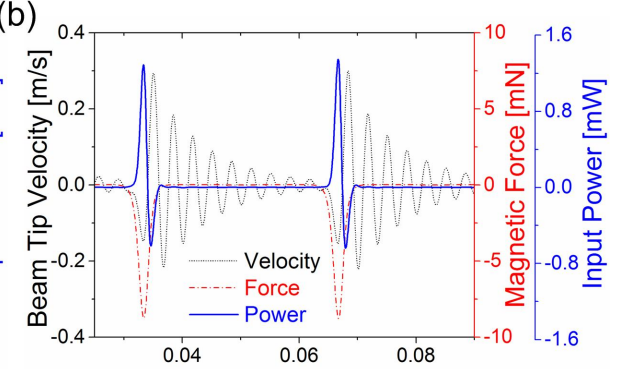

(d)

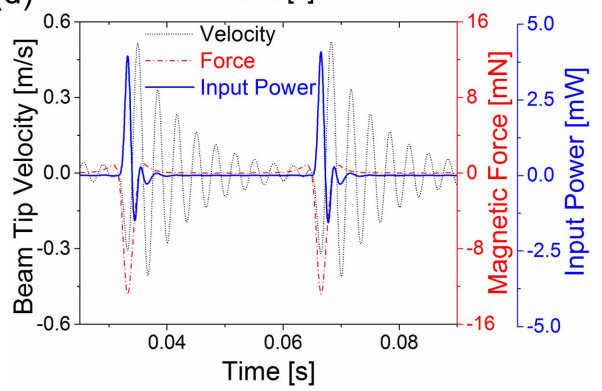

Figure 5: Beam tip velocity, magnetic force in the beam bending direction and beam input power for different arrangements, operating at $30 \mathrm{~Hz}$.

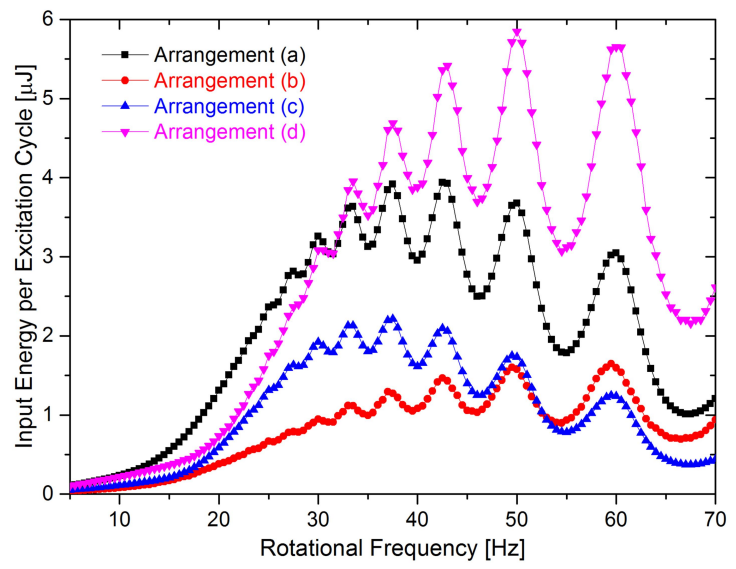

Figure 6: Input energy to the piezoelectric beam per excitation cycle for different arrangements of magnetic coupling.

has already rung down before the second excitation comes, but at high frequency ( $60 \mathrm{~Hz}$ in Fig. 7(b)), the beam is still vibrating when the second excitation comes. When the beam vibrating direction and the magnetic rotation direction are in the same direction, the input energy is enhanced, but when their motions are opposite, the input energy is decreased. $30 \mathrm{~Hz}$ (i.e. $\mathrm{T} \approx 33 \mathrm{~ms}$ ) corresponds to the duration of the ring-down process.

In order to determine the best arrangement of the magnetic coupling for the piezoelectric rotational energy harvester, the average input power is calculated, as shown in Fig. 8(a). Arrangement (a) also has the highest average input power at frequencies below $30 \mathrm{~Hz}$ and the average input power of arrangement (d) surpasses that of arrangement (a) when the frequency is over $30 \mathrm{~Hz}$, and the advantage of arrangement (d) is more evident at high frequencies.

In terms of the magnetic force, it can be attractive or repulsive. Different directions of the force also affect the dynamics of the piezoelectric beam and the performance of power extraction. In the following analysis, different directions of the magnetic force for arrangement (a) and arrangement (d) are considered. Fig. 8(b) illustrates the average input power to the piezoelectric beam against rotational frequency for different configurations. For arrangement (d), when the magnetic force is attractive, the input power is always much higher than that of other configurations. 

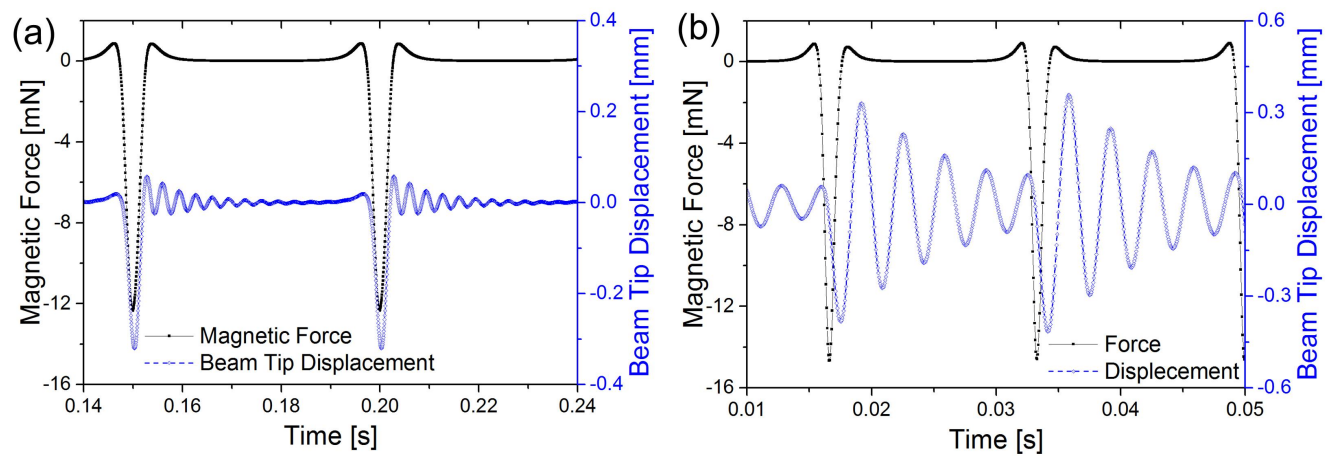

Figure 7: Magnetic force and beam tip displacement of arrangement (d) at different excitation frequencies, showing the interaction between the beam vibrations between two excitation cycles. (a) $20 \mathrm{~Hz}$ and (b) $60 \mathrm{~Hz}$.

However, there is a drawback to this configuration. As shown in Fig. 3(d), when the magnetic force is attractive, the beam is pulled towards the driving magnet initially. Then the gap between the magnets is reduced. As a result, the attractive force is stronger. In some case, there is a risk of collision between the driving magnet and the tip magnet, which has a negative effect on the reliability of the system. Therefore, some protection mechanism should be designed before using this arrangement, which might increase the complexity of the harvester. For arrangement (a), the possibility of collision is eliminated by maintaining the piezoelectric beam and the driving magnet moving in two parallel planes.
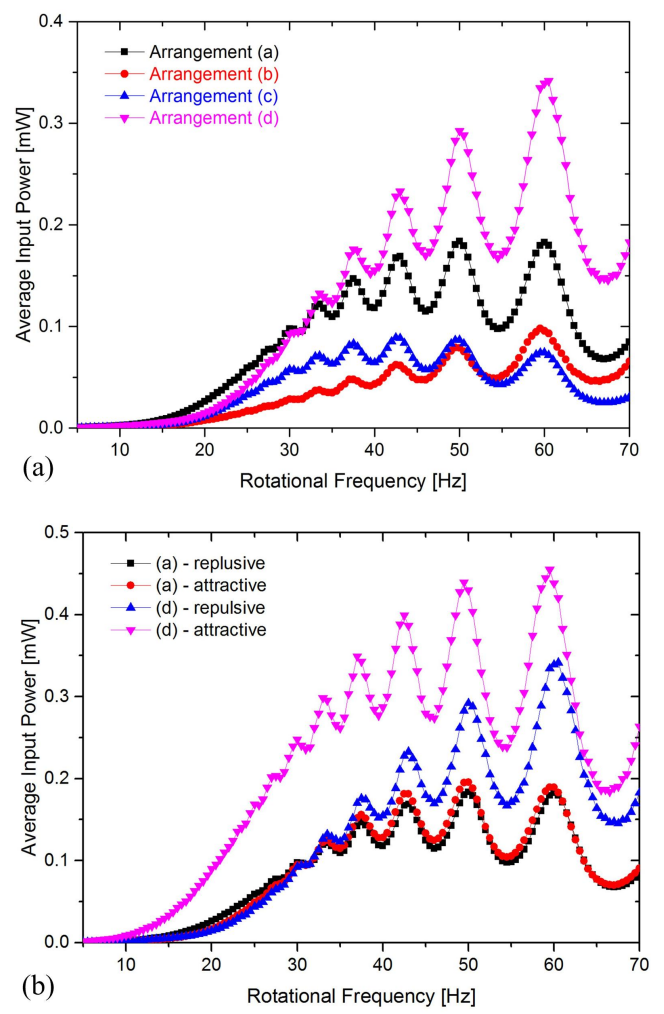

Figure 8: (a) Average input power to the piezoelectric beam for different arrangements of magnetic coupling versus frequency of the rotating magnet and (b) average input power for the attractive and the repulsive orientations for arrangement (a) and (d). 


\subsection{Electrical behaviours of the harvester}

The mechanical strain in the cantilever beam is converted to electrical energy by the piezoelectric effect. The conversion is considered theoretically in Eq. 5 and Eq. 6. Using the above theories, the electrical behaviour of the harvester is studied for arrangement (a)-repulsive. Fig. 9 shows the output voltage of the beam on a $100 \mathrm{k} \Omega$ load under magnetic plucking. The rotational frequency of the driving magnet is $20 \mathrm{~Hz}(50 \mathrm{~ms})$, whereas the beam vibrates at approximately $300 \mathrm{~Hz}(3.35 \mathrm{~ms})$, which is its natural frequency $\left(f_{n}\right)$. Frequency up-conversion is, therefore, achieved by this plucking mechanism, allowing the device to harvester low speed rotational energy with a broad bandwidth.

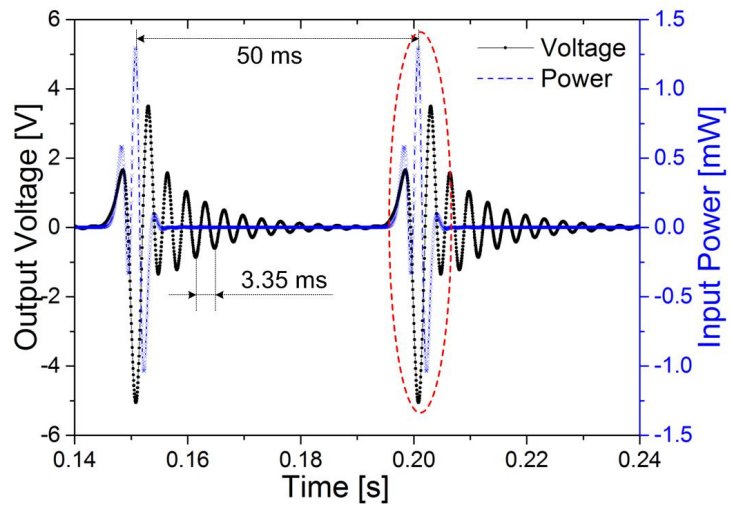

Figure 9: Output voltage of the piezoelectric beam under magnetic plucking, showing the implementation of frequency up-conversion.

Another special behaviour of the harvester is the ring-down pattern. For the first four peaks (shown in the red circle in Fig. 9) after the initial plucking, they are not typical damped free vibrations, as the driving magnet still affects the tip magnet during this period. The input power of the driving magnet varies with time, as shown in the figure. After that period, there is no interaction between the magnets, and the vibration is then in a damped-free-vibration form.

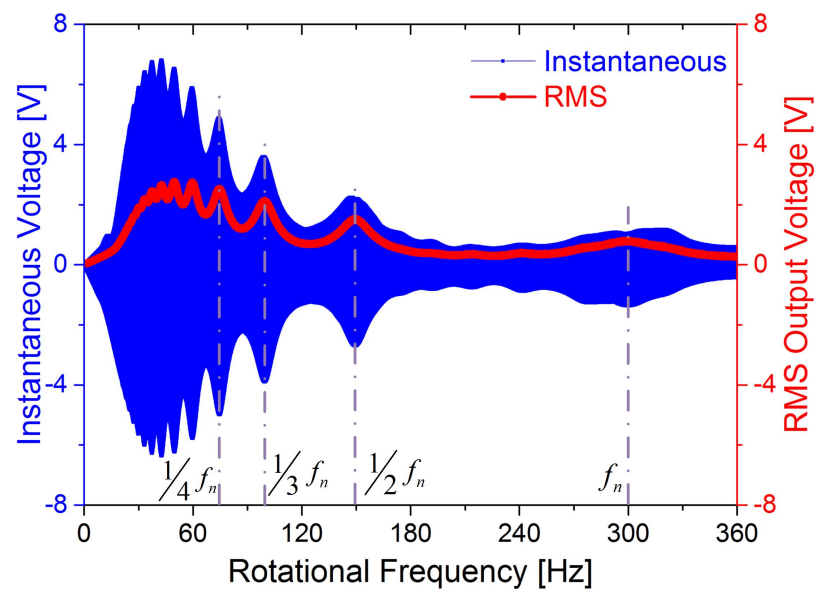

Figure 10: Instantaneous and RMS output voltage on a $100 \mathrm{k} \Omega$ load against rotational frequency of driving magnet for arrangement (a)-repulsive.

In order to understand the behaviour of the piezoelectric beam under different rotational frequencies, a frequency sweep is presented in Fig. 10. This figure shows the instantaneous and RMS output voltage versus rotational frequency of the driving magnet. The rotational frequency of the driving magnet is increased with a constant acceleration against time. The acceleration is small enough to ensure the accuracy of the response for specific frequencies.

Fluctuation of the output voltage is also exhibited in this figure. The reason has been shown in Fig. 7. There is a relationship between the frequency of each peak and the natural frequency $\left(f_{n}\right)$ of the beam. For ease of discussion, 
the peaks from right to left in Fig. 10 are called the first peak, the second peak, etc.. The frequency of the $i^{\text {th }}$ peak can be expressed as

$$
f_{i}=\frac{f_{n}}{n \cdot i},
$$

where $\mathrm{n}$ is the number of driving magnets on the revolving host.

The output voltage at the natural frequency is not the highest, as shown in Fig. 10. This is caused by the complicated form of the magnetic force for arrangement (a) (shown in Fig. 4). In addition, the RMS output voltages at low frequencies are reasonable high, which shows the device has a broad bandwidth at low frequencies.

\section{Experimental Validation}

\subsection{Experimental set-up}

In order to verify the accuracy of the theoretical model, an experimental validation was carried out. The experimental set-up is demonstrated in Fig. 11. The piezoelectric beam is clamped on a beam holder at one end. The holder is installed on an adjustable platform which is capable of precisely controlling the position of the piezoelectric beam in three directions. A high speed stepper motor (Phidgets 3303) with the step angle of $1.8^{\circ}$ is placed underneath the beam with a revolving plate mounting on the motor's shaft. The motor is driven by a bipolar motor control circuit (Phidgets 1067) which has a position resolution of $1 / 16$ step. The acceleration is also programmable to achieve any desired rotational speed. The maximum achievable speed is $4800 \mathrm{rpm}$ (or $80 \mathrm{~Hz}$ ), which is not usually attainable using stepper motors. A magnet is mounted on the revolving plate as the driving magnet. Magnet plucking is formed by the driving magnet and the tip magnet at the beam's free end. The relative position of the magnets is regulated by the adjustable platform. A laser displacement sensor is also adopted in the experiment to measure the vibration of the piezoelectric beam. The output of the piezoelectric beam is directly connected with a $100 \mathrm{k} \Omega$ resistive load. In this experiment, arrangement (a)-repulsive was used due to its advantage of high output power and no potential risk of collision between the magnets. The parameters of the harvester are the same as those listed in Table 1.

In this experiment, due to the high driving torque of the stepper motor, the rotor's motion is not affected by the interaction of the magnets. Therefore, the instantaneous velocity of the rotor is unaffected by the magnetic force, whereas in some cases, such as miniature turbines, the torque provided by the rotating host is limited. In this case, the instantaneous velocity of the rotor is affected by the subsequent transduction methods, and the rotational velocity is not constant within one rotation.

The experimental set-up was well designed. The factors that might cause experimental errors were eliminated in the design process. Therefore, data repeatability is not a significant issue in this experiment. Still, the possible errors could be from the variation of the magnetic plucking force, variation of the clamping condition of the beam or the inaccuracy of the driving frequency of the motor. These potential issues were addressed respectively in the design process.

The magnetic plucking force is determined by the relative position between the driving magnet and the tip magnet. The relative position remained the same for each plucking by rigidly fixing the motor and the piezoelectric beam on the same anti-vibration test bench. The beam was firmly clamped on a platform and the clamping condition was not changed during the whole test. For driving frequency of the motor, the rotating speed was controlled by the control circuit. The rotating frequency can be accurately adjusted as the experiment requires.

\subsection{Design of experiments}

This work aimed to implement the rotational harvester design and to verify the electromechanical dynamics found in the theoretical study. Therefore, two experimental tests were designed and conducted.

The first test was to examine the capability of the theoretical model in predicting the performance of the harvester. Fig. 12 shows the output voltage and tip displacement of the harvester operating at the rotating frequency of $30 \mathrm{~Hz}$. Similar fitting between the theoretical prediction and experimental results can be obtained as well for different driving frequencies. For simplicity, only $30 \mathrm{~Hz}$ is illustrated in this paper. The second test was designed to examine the bandwidth and to verify the theoretically proposed relationship between the frequency and the peak output power. Therefore, in this test a frequency sweep was conducted. The rotating frequency of the rotor increased with a constant 


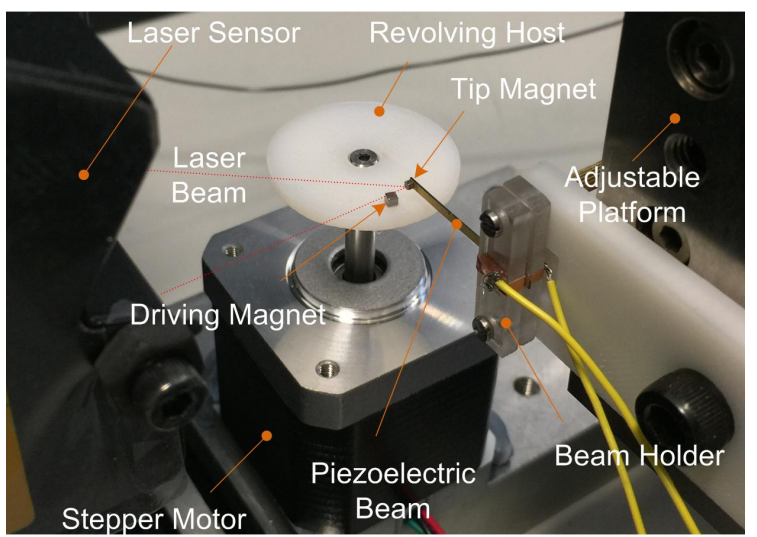

Figure 11: Experimental set-up of the rotational energy harvester for the arrangement of (a)-repulsive.

angular acceleration $\left(0.16 \mathrm{r} / \mathrm{s}^{2}\right)$. Both of these tests also demonstrate the advantages of the broadband rotational energy harvester design.

Since the purpose of the experiments is to validate the general trend and form of behaviour predicted by the model rather than the models precision, experimental precision was not quantitatively estimated. Likely sources of errors include the variation of the magnetic plucking force, instability of the beam clamping condition or inaccuracy of the motor driving frequency. These potential issues have already considered and addressed via design process respectively.

\subsection{Results and discussion}

Fig. 12 shows the theoretical and experimental tip displacement and output voltage of the rotational energy harvester. In this comparison, the stepper motor rotates at $30 \mathrm{~Hz}$ and the resonant frequency $\left(f_{n}\right)$ of the piezoelectric beam is about $300 \mathrm{~Hz}$. It is shown that the tip displacement and voltage fit well between the theoretical simulation and the experimental results in terms of amplitude, frequency and damping. The mismatching at the beginning of each plucking could be caused by the phase difference generated in the experiment. Overall, this comparison indicates the capability of the theoretical model in analysing the performance of the harvester.

Then, a frequency sweep analysis was conducted to examine the behaviour of the harvester under different rotational speeds. In the experiment, the motor rotated from static to $55 \mathrm{~Hz}$ with a constant acceleration. The instantaneous output voltage and RMS voltage against rotational frequency of the driving magnet is illustrated in Fig. 13. Fluctuation of the output voltage can be observed as well from both the instantaneous and RMS voltage in experimental results. The frequencies of the peaks from right to left are $50 \mathrm{~Hz}, 42.5 \mathrm{~Hz}, 37.5 \mathrm{~Hz}$, etc. They are $1 / n$ of the natural frequency of the beam $(n=6,7,8, \ldots)$. The result verifies the conclusion made in Eq. 8. From the RMS output voltage, it is seen that the system has a wide bandwidth at low frequency with high power output. The RMS voltage is higher than $1.5 \mathrm{~V}$ between $15 \mathrm{~Hz}$ to $35 \mathrm{~Hz}$, so that the output power is more than $20 \mu \mathrm{W}$ in this range.

\section{Conclusion}

In this paper, a methodology for low-speed broadband rotational energy harvesting is presented. Frequency upconversion, a mechanism to convert low rotational frequency to high vibration frequency, is implemented by magnetic plucking of the piezoelectric beam. Two magnets, installed on a revolving host and the free end of the piezoelectric beam, are employed in the harvester to facilitate the magnetic plucking. The non-contact plucking mechanism improves the reliability of the system.

A theoretical model was established based on the distributed-parameter method to analyse and optimize the harvester. Different configurations of harvesters in terms of beam bending direction, magnetization, magnet rotation direction and initial relative position of the driving magnet were considered. The arrangement of (a)-repulsive was chosen as the best due to its merits of high output power and no potential collision of the magnets. The electrical 

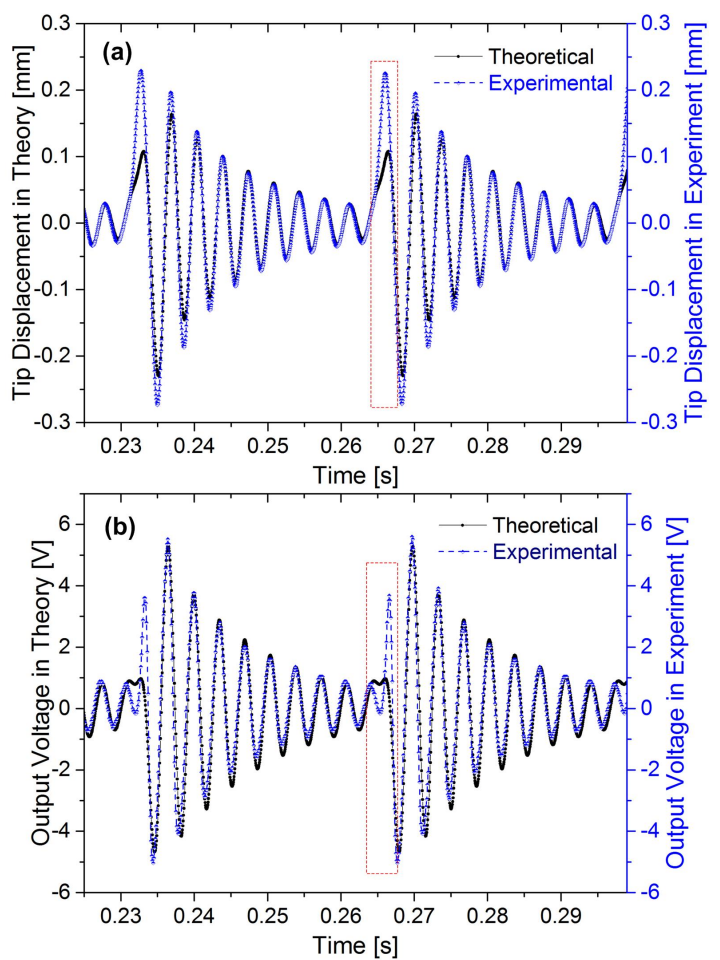

Figure 12: Comparison of the electromechanical behaviours of the harvester acquired in theory and in experiment, showing the accuracy of the theoretical model. (a) Comparison of the tip displacement and (b) comparison of the output voltage.

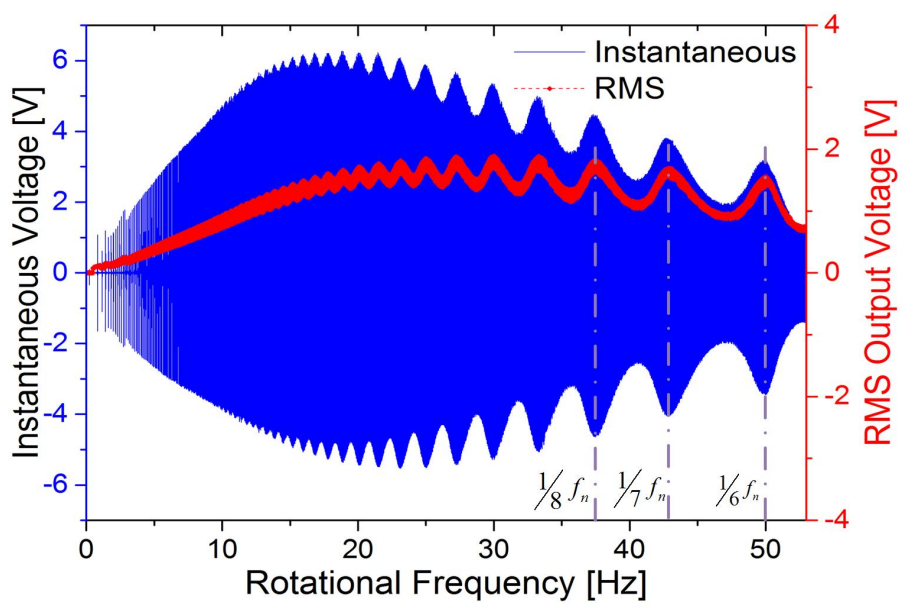

Figure 13: Instantaneous and RMS output voltage of the harvester for a $100 \mathrm{k} \Omega$ resistive load versus rotational frequency of the driving magnet.

behaviours of the harvesters were also examined. The analysis shows that the harvester has a wide bandwidth at low rotational speeds and the ripples at high frequencies are related to the beam's natural frequency.

An experimental validation was carried out to verify the theoretical model. The experimental results fit well with the theoretical analysis. The harvester had more than $20 \mu \mathrm{W}$ output power in the rotational frequency range from 15 $\mathrm{Hz}$ to $35 \mathrm{~Hz}$. This rotational harvester has the advantage of compactness, simplicity in structure, high reliability (due to the non-contact plucking) and wide operating bandwidth at low frequency. It can be used in the scenarios of tire condition monitoring, flow energy harvesting using turbines and condition monitoring of rotating machinery. 


\section{Acknowledgement}

The work is supported by the China Scholarship Council (CSC) and the Department of Electrical and Electronic Engineering, Imperial College London. We would also like to thank Prof. Andrew Holmes for use of apparatus.

\section{References}

[1] T. Krupenkin, J. A. Taylor, Reverse electrowetting as a new approach to high-power energy harvesting, Nature Communications 2 (2011) 448.

[2] E. M. Yeatman, Energy harvesting from motion using rotating and gyroscopic proof masses, Proceedings of the Institution of Mechanical Engineers, Part C: Journal of Mechanical Engineering Science 222 (1) (2008) 27-36

[3] M. Gorlatova, A. Wallwater, G. Zussman, Networking low-power energy harvesting devices: Measurements and algorithms, IEEE Transactions on Mobile Computing 12 (9) (2013) 1853-1865.

[4] S. P. Beeby, M. J. Tudor, N. M. White, Energy harvesting vibration sources for microsystems applications, Measurement Science and Technology 17 (12) (2006) R175.

[5] P. D. Mitcheson, E. M. Yeatman, G. K. Rao, A. S. Holmes, T. C. Green, Energy harvesting from human and machine motion for wireless electronic devices, Proceedings of the IEEE 96 (9) (2008) 1457-1486.

[6] E. Halvorsen, Fundamental issues in nonlinear wideband-vibration energy harvesting, Physical Review E 87 (2013) 042129.

[7] M. F. Daqaq, R. Masana, A. Erturk, D. Dane Quinn, On the role of nonlinearities in vibratory energy harvesting: A critical review and discussion, Applied Mechanics Reviews 66 (4) (2014) 040801-040801.

[8] C. R. Bowen, H. A. Kim, P. M. Weaver, S. Dunn, Piezoelectric and ferroelectric materials and structures for energy harvesting applications, Energy Environ. Sci. 7 (2014) 25-44.

[9] E. R. Westby, E. Halvorsen, Design and modeling of a patterned-electret-based energy harvester for tire pressure monitoring systems, IEEE/ASME Transactions on Mechatronics 17 (5) (2012) 995-1005.

[10] C. R. Bowen, M. H. Arafa, Energy harvesting technologies for tire pressure monitoring systems, Advanced Energy Materials 5 (7), 1401787.

[11] Y.-J. Wang, C.-D. Chen, C.-K. Sung, System design of a weighted-pendulum-type electromagnetic generator for harvesting energy from a rotating wheel, IEEE/ASME Transactions on Mechatronics 18 (2) (2013) 754-763

[12] M. Guan, W.-H. Liao, Design and analysis of a piezoelectric energy harvester for rotational motion system, Energy Conversion and Management 111 (2016) $239-244$

[13] H. Fu, E. M. Yeatman, A miniature radial-flow wind turbine using piezoelectric transducers and magnetic excitation, Journal of Physics: Conference Series 660 (1) (2015) 012058.

[14] R. Riemer, A. Shapiro, Biomechanical energy harvesting from human motion: theory, state of the art, design guidelines, and future directions, Journal of NeuroEngineering and Rehabilitation 8 (1) (2011) 1 - 13.

[15] P. Pillatsch, E. M. Yeatman, A. S. Holmes, A piezoelectric frequency up-converting energy harvester with rotating proof mass for human body applications, Sensors and Actuators A: Physical 206 (2014) 178 - 185.

[16] G. Manla, N. M. White, M. J. Tudor, Numerical model of a non-contact piezoelectric energy harvester for rotating objects, IEEE Sensors Journal 12 (6) (2012) 1785-1793.

[17] L. Gu, C. Livermore, Compact passively self-tuning energy harvesting for rotating applications, Smart Materials and Structures 21 (1) (2012) 015002.

[18] H. Fu, E. M. Yeatman, A miniaturized piezoelectric turbine with self-regulation for increased air speed range, Applied Physics Letters 107 (24) (2015) 243905.

[19] S. Roundy, J. Tola, Energy harvester for rotating environments using offset pendulum and nonlinear dynamics, Smart Materials and Structures 23 (10) (2014) 105004

[20] T. T. Toh, P. D. Mitcheson, A. S. Holmes, E. M. Yeatman, A continuously rotating energy harvester with maximum power point tracking, Journal of Micromechanics and Microengineering 18 (10) (2008) 104008.

[21] M. Perez, S. Boisseau, P. Gasnier, J. Willemin, M. Geisler, J. L. Reboud, A cm scale electret-based electrostatic wind turbine for low-speed energy harvesting applications, Smart Materials and Structures 25 (4) (2016) 045015.

[22] H. Fu, R. Xu, K. Seto, E. M. Yeatman, S. G. Kim, Energy harvesting from human motion using footstep-induced airflow, Journal of Physics: Conference Series 660 (1) (2015) 012060.

[23] D. Zhao, C. Ji, C. Teo, S. Li, Performance of small-scale bladeless electromagnetic energy harvesters driven by water or air, Energy 74 (2014) $99-108$.

[24] S. Roundy, P. K. Wright, A piezoelectric vibration based generator for wireless electronics, Smart Materials and Structures 13 (5) (2004) 1131.

[25] Y. Suzuki, D. Miki, M. Edamoto, M. Honzumi, A mems electret generator with electrostatic levitation for vibration-driven energy-harvesting applications, Journal of Micromechanics and Microengineering 20 (10) (2010) 104002.

[26] P. Pillatsch, N. Shashoua, A. S. Holmes, E. M. Yeatman, P. K. Wright, Degradation of piezoelectric materials for energy harvesting applications, Journal of Physics: Conference Series 557 (1) (2014) 012129.

[27] D. A. Howey, A. Bansal, A. S. Holmes, Design and performance of a centimetre-scale shrouded wind turbine for energy harvesting, Smart Materials and Structures 20 (8) (2011) 085021

[28] F. Xu, F. Yuan, J. Hu, Y. Qiu, Miniature horizontal axis wind turbine system for multipurpose application, Energy 75 (2014) 216 - 224.

[29] N. Rezaei-Hosseinabadi, A. Tabesh, R. Dehghani, A topology and design optimization method for wideband piezoelectric wind energy harvesters, IEEE Transactions on Industrial Electronics 63 (4) (2016) 2165-2173.

[30] Y. Yang, Q. Shen, J. Jin, Y. Wang, W. Qian, D. Yuan, Rotational piezoelectric wind energy harvesting using impact-induced resonance, Applied Physics Letters 105 (5). 
[31] S. Priya, Modeling of electric energy harvesting using piezoelectric windmill, Applied Physics Letters 87 (18).

[32] F. Goldschmidtboeing, P. Woias, Characterization of different beam shapes for piezoelectric energy harvesting, Journal of Micromechanics and Microengineering 18 (10) (2008) 104013.

[33] S. Saadon, O. Sidek, A review of vibration-based \{MEMS\} piezoelectric energy harvesters, Energy Conversion and Management 52 (1) (2011) $500-504$.

[34] H. Kulah, K. Najafi, Energy scavenging from low-frequency vibrations by using frequency up-conversion for wireless sensor applications, IEEE Sensors Journal 8 (3).

[35] Design and development of a multipurpose piezoelectric energy harvester, Energy Conversion and Management 96 (2015) 430 - 439.

[36] G. Akoun, J. P. Yonnet, 3d analytical calculation of the forces exerted between two cuboidal magnets, IEEE Transactions on Magnetics 20 (5) (1984) 1962-1964.

[37] N. E. Dutoit, B. L. Wardle, S.-G. Kim, Design considerations for mems-scale piezoelectric mechanical vibration energy harvesters, Integrated Ferroelectrics 71 (1) (2005) 121-160.

[38] A. Erturk, D. J. Inman, Base Excitation Problem for Cantilevered Structures and Correction of the Lumped-Parameter Electromechanical Model, John Wiley \& Sons, Ltd, 2011, pp. 19-48.

[39] D. J. Inman, Engineering vibration, Prentice Hall, Englewood Cliffs, NJ, 2007.

[40] A. Erturk, D. J. Inman, An experimentally validated bimorph cantilever model for piezoelectric energy harvesting from base excitations, Smart Materials and Structures 18 (2) (2009) 025009. 